\title{
Constructing Complete Radio Frequency Receiver for LTE TDD Transceiver
}

\author{
Dalia Sadek \\ Ain shams university \\ Cairo - Egypt
}

\author{
Heba A. Shawkey \\ Electronics Research Institute \\ (ERI) \\ Giza - Egypt
}

\author{
Abdelhalim Zekry \\ Ain shams university \\ Cairo - Egypt
}

\begin{abstract}
In this paper, the design, implementation and testing of the Zero IF receiver for Long Term Evolution (LTE) band 36 extending from $1930 \mathrm{MHz}$ to $1990 \mathrm{MHz}$ for TDD mode of transmission. Comprising a passive part and active part. The passive part consists of a microstrip patch antenna and a Hairpin BPF filter which are fabricated with a total area of $43 \times 36 \mathrm{~mm}^{2}$ for the antenna and $38.6 \times 32.16 \mathrm{~mm}^{2}$ for the filter. The proposed devices are fabricated using FR-4 material substrate with dielectric constant of $\mathrm{Er}=4.4$, thickens $\mathrm{h}=1.6$, and Loss tangent of 0.025 .
\end{abstract}

The active part includes Low noise amplifier, IQ demodulator and frequency synthesizer. Which are a real made component selected off-shelf to satisfy the standards for LTE receiver characteristics.

The proposed RF front-end receiver achieves a $4.022 \mathrm{~dB}$ NF, $2.65 \mathrm{dBm}$ IIP3.

\section{Keywords}

LTE, TDD, band 36, Zero IF receiver, Micro-strip,

Patch antenna, filter, FR-4 material, LNA, Frequency Synthesizer and Demodulator

\section{INTRODUCTION}

LTE is the abbreviation for Long Term Evolution [1] and is the trademark owned by ETSI (European Telecommunications Standards Institute) for the wireless data communications technology and a development of the GSM/UMTS standards. However, many companies do play an active role in the LTE project. A new DSP (digital signal processing) techniques and modulations that were developed around the turn of the millennium were used to increase the capacity and speed of wireless data network which is the main goal of the LTE. An additional goal was the redesign and simplification of the network architecture to an IP-based system with significantly reduced transfer latency compared to the $3 \mathrm{G}$ architecture [2]. The downlink capacity of at least $300 \mathrm{Mbps}$ and uplink capacity of at least 75Mbps.

LTE supports both frequency division duplex (FDD) and time-division duplex (TDD). The LTE can use QPSK, 16QAM or 64QAM modulation schemes. It supports six different channel bandwidths: $1.4 \mathrm{MHz}, 3 \mathrm{MHz}, 5 \mathrm{MHz}$, $10 \mathrm{MHz}, 15 \mathrm{MHz}$, and $20 \mathrm{MHz}$ [3].

In that respect are several existing plans and implementations that have been described in the literature. Such as 1900 Direct Conversion Receiver using 0.13um CMOS in [4]. A front-end for LTE direct conversion SAW-less receiver using
A 1.2-V CMOS in [5]. A WiMAX/LTE receiver front-end using $90 \mathrm{NM}$ CMOS in [6]. Based on our knowledge, it is the first time to introduce a design and implementation of the whole LTE RF receiver which consists of an antenna, band pass filter, Low noise amplifier, IQ demodulator, and frequency synthesizer for LTE band 2 FDD or band 36 TDD.

The paper is organized as follows; section 2 presents the receiver architecture and key parameters, section 3 the passive front end for LTE receiver, section 4 the active front end for the LTE receiver section 5 measurements of the whole RF receiver finally, section 6 is for conclusion.

\section{RECEIVER ARCHITECTURES AND KEY PARAMETERS}

The function of a receiver is to successfully demodulate a desired signal in the presence of strong interference and noise [7].

A receiver system needs to minimize cost and power consumption a number of different requirements translate into different receiver architectures. The development towards small size and low cost integrated circuits with a higher degree of components, integrated on the chip has made the simple architecture of the Zero IF receiver architectures. The proposed $\mathrm{RF}$ receiver (Zero IF architecture) shown in figure 1.

The main parts of the RF receiver are the following:

1. Antenna: E-Shape patch antenna operates 1903.2 $\mathrm{MHz}$ to $2011 \mathrm{MHz}, \mathrm{BW} 107.7 \mathrm{MHz}$, at return loss $6 \mathrm{~dB}$, minimum return loss $-28.58 \mathrm{~dB}$ this antenna covers LTE TDD frequency band 36.

2. Band-pass filter (BPF): The designed and implemented Hairpin filter operates at the center frequency $1950 \mathrm{MHz}$ and $90 \mathrm{MHz} \mathrm{BW}$ at $3 \mathrm{~dB}$.

3. Low-noise amplifier (LNA): The selected RF Low Noise Amplifier (RF2442) has Low Noise, High Intercept and $500 \mathrm{MHz}$ to $2500 \mathrm{MHz}$ Operation. The LNA achieves gain of about $12 \mathrm{~dB}$ at the operating frequency $1.95 \mathrm{GHz}$ and $\mathrm{Vcc}=3.6 \mathrm{~V}$.

4. Local oscillator (LO): The frequency synthesizer is selected (LTC 6949) with step size of $200 \mathrm{KHz}$ and frequency range from $0.37 \mathrm{GHz}$ to $5.7 \mathrm{GHz}$, so that it covers all LTE bands.

5. The mixer: The selected direct conversion $\mathrm{I} / \mathrm{Q}$ demodulator (LTC 5585) has a frequency range from $0.7 \mathrm{GHz}$ to $3 \mathrm{GHz}$. The $\mathrm{RF}$ signal is directly converted into I and Q baseband signals. With 
The key parameters to measure for the receiver are the receiver Noise Figure, The receiver sensitivity, the

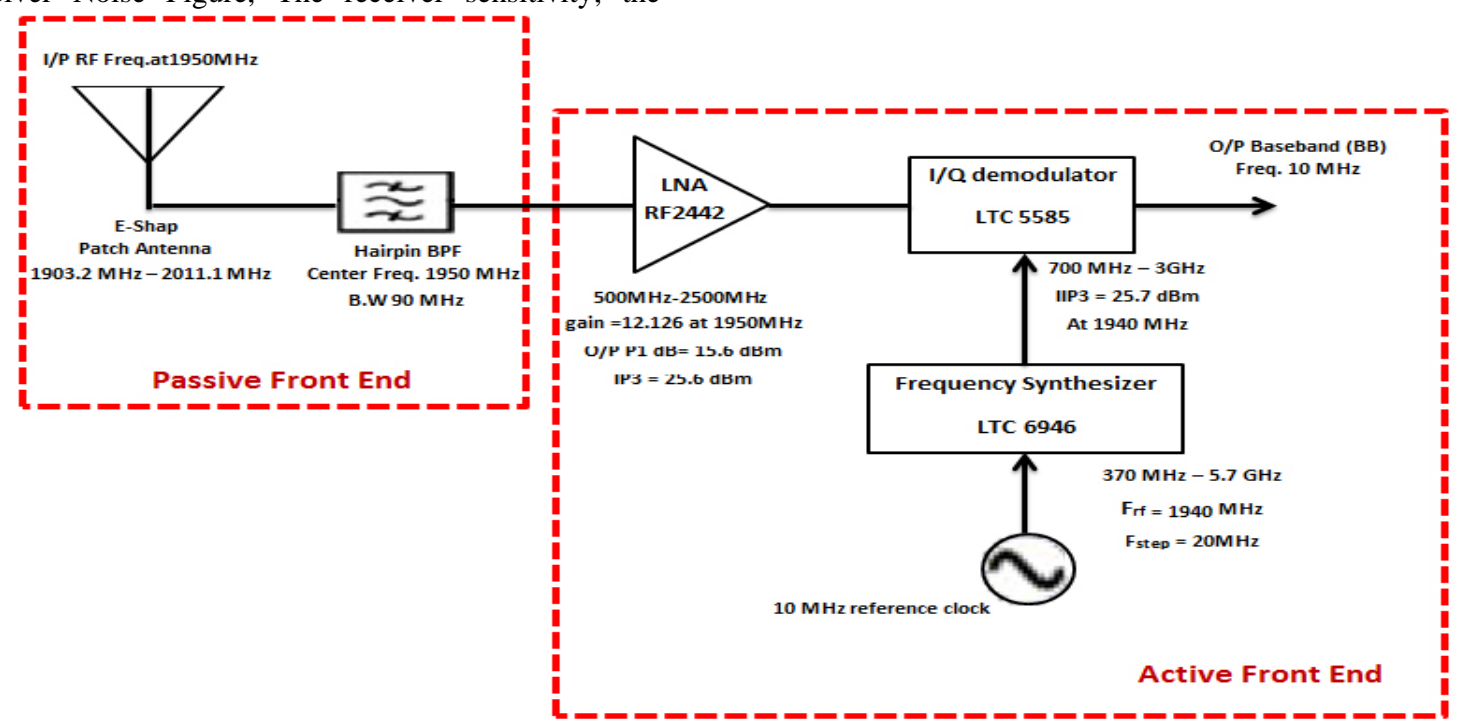

Fig 1: The proposed zero if receiver topology

\section{PASSIVE FRONT END FOR LTE RECEIVER}

Micro-strip technics are used for the passive part (the antenna and the band pass filter). The detailed description passive front end is given in [8]

\subsection{LTE Antenna Design}

Figure 2 (a) and (b) shows the top and bottom views of the design of the proposed antenna whose dimensions are determined with the help of CST simulator and listed in Table 1 [8].

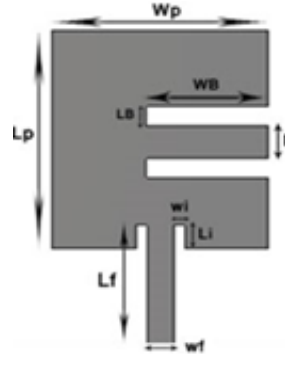

(a)

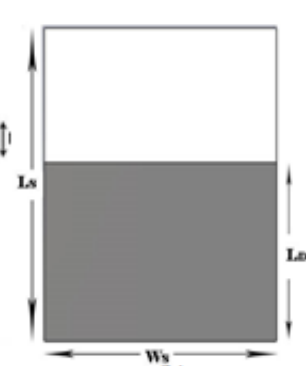

(b)
Fig 2: Antenna Design (a) Top view (b) Bottom view

Table 1. Antenna parameter's size

\begin{tabular}{|c|c|c|c|}
\hline Parameter & Size(mm) & Parameter & Size(mm) \\
\hline $\mathrm{L}_{\mathrm{s}}$ & 43 & $\mathrm{~L}_{\mathrm{D}}$ & 28 \\
\hline $\mathrm{W}_{\mathrm{s}}$ & 36 & $\mathrm{Li}$ & 6.6 \\
\hline $\mathrm{Lp}$ & 27.5 & $\mathrm{Wi}$ & 1.25 \\
\hline $\mathrm{W}_{\mathrm{p}}$ & 24 & $\mathrm{~W}_{\mathrm{B}}$ & 13.57 \\
\hline $\mathrm{L}_{\mathrm{f}}$ & 18.5 & $\mathrm{~L}_{\mathrm{B}}$ & 2.45 \\
\hline $\mathrm{W}_{\mathrm{f}}$ & 2.97 & $\mathrm{~L}_{\mathrm{A}}$ & 4.05 \\
\hline
\end{tabular}

The antenna is then fabricated and its photographs are shown in Figure 3 (a) and (b)

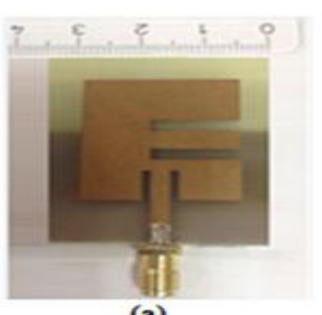

(a)

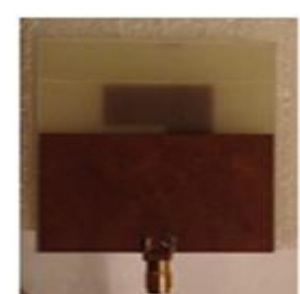

(b)
Fig 3: Antenna photography (a) Top view (b) Bottom view

Figure 4 demonstrates the return loss (S-Parameter) value, as a result of antenna simulation and antenna measurement. From the antenna simulation the antenna operates between 1903.3 MHz and 2011.1 MHz with Bandwidth 107.7 MHz at return loss $-6 \mathrm{~dB}$, minimum return loss $-28.58 \mathrm{~dB}$ this antenna covers LTE TDD frequency band 36 [8].

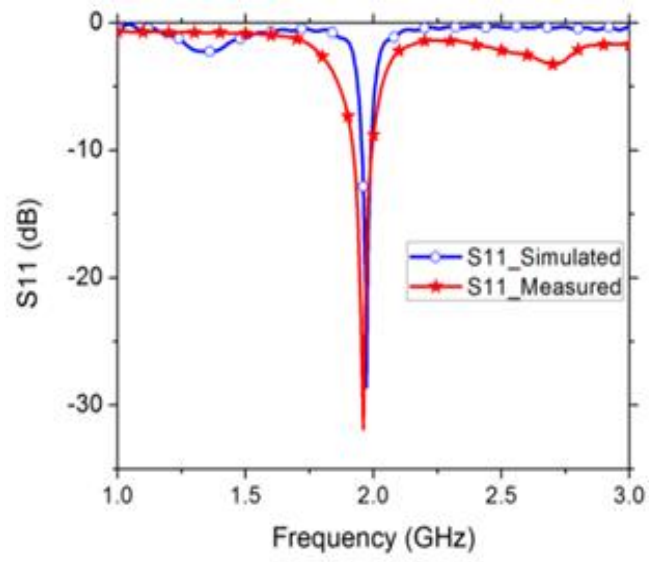

Fig 4: Comparison between the simulation and measured 
results for the antenna

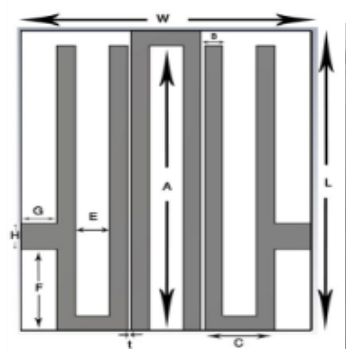

(a)

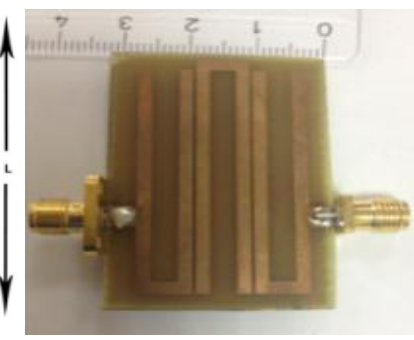

(b)
Fig 5: Filter Design (a) Top view (b) Filter photography

\subsection{LTE Filter Design}

The filter is designed and simulated using the CST simulator program Figure 5 (a) and (b) shows Filter design Top view and the photograph of its fabrication. The dimensions are given in Table 2 [8].

Table 2. Filter parameter's size

\begin{tabular}{|c|c|c|c|}
\hline Parameter & Size(mm) & Parameter & Size(mm) \\
\hline L & 38.6 & E & 3.8 \\
\hline W & 32.16 & H & 3.2 \\
\hline A & 36.6 & G & 4 \\
\hline B & 2 & F & 11.2 \\
\hline C & 7.8 & t & 0.38 \\
\hline
\end{tabular}

The simulated performance of the filter is shown in Figure 6 . From the measurements, the $-3 \mathrm{~dB} \mathrm{BW}$ of the filter amounts to $90 \mathrm{MHZ}$ and a center frequency of $1950 \mathrm{MHz}$. It is clear from the figure that there is a satisfactory agreement between the simulated and measured results [8].

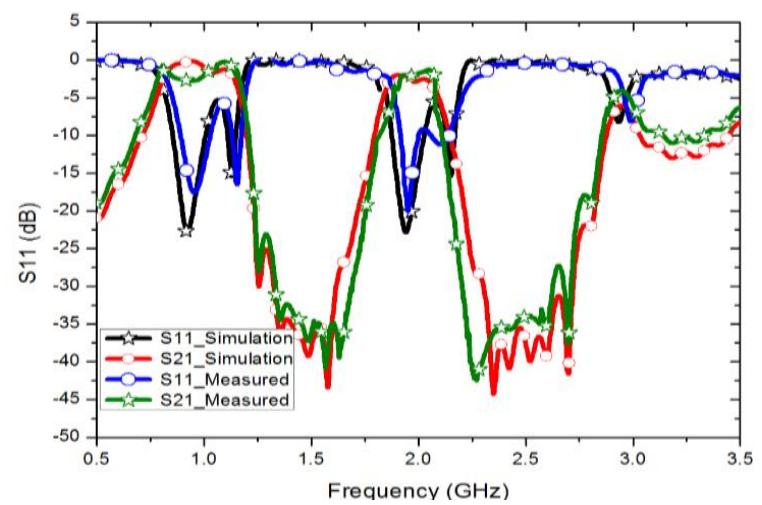

Fig 6: Comparison between the simulation and measured results for the Hairpin filter

\section{ACTIVE FRONT END}

The active components of Zero IF Architectures consist of Low noise amplifier, demodulator and synthesizer, which are real made components were selected off-shelf from different types and different companies to satisfy the specifications required for the proposed LTE front end receiver. Here also, their performance parameters are verified by intensive RF measurements.

\subsection{Low Noise Amplifier (LNA)}

The first component in the active front end is the LNA. It amplifies the received signals to acceptable levels with minimum self-generated additional noise. It is usually located very close to the Antenna [9] to reduce losses in the feed-line. LNA is a design that minimizes the noise figure of the system by matching the device to its noise matching impedance [10].

RF2442 is the selected low noise amplifier. It has a very high dynamic range designed for front end receiver for digital cellular applications and covers all bands of LTE from 500 $\mathrm{MHz}$ to $2500 \mathrm{MHz}$. According to the data sheet, its performance parameters are outlined as follows:

The DC power consumption is a major parameter in the LNA. RF2442 operates from $3.6 \mathrm{~V}$ power supply and consumes about $0.23 \mathrm{~W}$. The measured S-parameters of the amplifier as a function of frequency is shown in Fig. 7. The frequency range is that of LTE from $1930 \mathrm{MHz}$ to1980MHz.

Gain $(\mathrm{dB})=10 \log ($ Pout $/$ Pin $)=$ S21 $(\mathrm{dB})$

It is clear from the figure that, at the center Frequency 1950 $\mathrm{MHz}$, the Gain S21=12.126 dB, the Return losses at the input and output respectively, $\mathrm{S} 11=-15 \mathrm{~dB}$, and $\mathrm{S} 22=-12.5 \mathrm{~dB}$, and the Reverse isolation $\mathrm{S} 12=-20 \mathrm{~dB}$

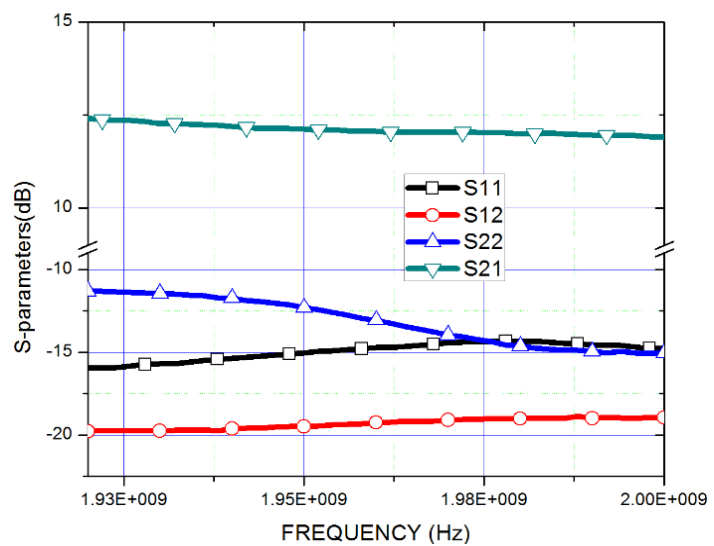

Fig 7: LNA S-parameters (dB) measurement

For the measurement of the one $\mathrm{dB}$ compression point $\mathrm{P} 1-\mathrm{dB}$ of the LNA, the input power is changed and the corresponding output power at $1950 \mathrm{MHz}$ is measured and the results are plotted in Figure 8.

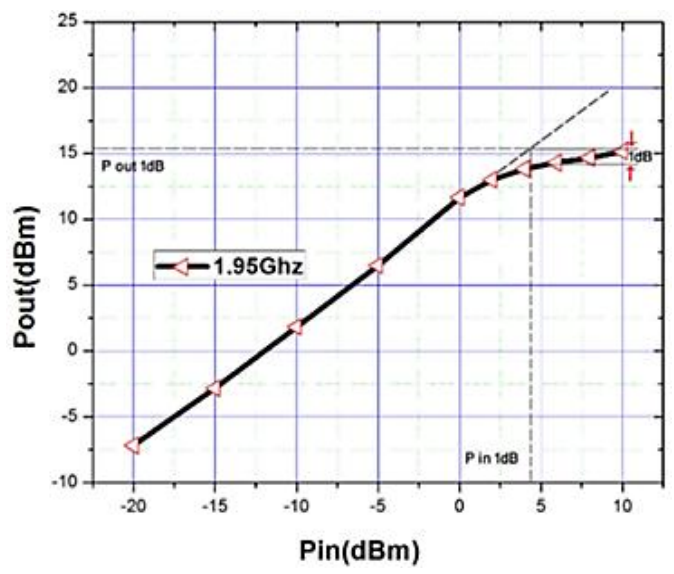

Fig 8: 1dB compression point at 1950MHz

From the curve, the Output $\mathrm{P} 1 \mathrm{~dB}=15.6 \mathrm{dBm}$. One can 
calculate the third intercept point of the amplifier IP3 use the formula: Output at IP3 output at P1dB + 11dB [11], which results in Output IP3 26.6dBm.

\subsection{Frequency Synthesizer}

The second component in the active front end is the frequency synthesizer. It is an electronic system for generating any of a range of frequencies from a single fixed time base or oscillator. A frequency synthesizer can combine frequency to produce the desired output signal [12].

The LTC6946 is the selected Frequency synthesizer for the proposed LTE RF receiver. It has high performance, low noise, phase-locked loop (PLL) with a fully integrated VCO, including a reference divider, phase-frequency detector (PFD) with phase-lock indicator, ultra-low noise charge pump, integer feedback divider, and VCO output divider. Figure 10 shows a simplified LTC6946 block diagram, with the external loop filter components. The phase/frequency detector (PFD) compares the phase and frequency of the reference clock $f_{R E F}$, after dividing it by $R$ to produce $f_{P F D}$, for those of the VCO following an integer division of N. The PFD, then controls the current sources of the charge pump to ensure that the VCO runs at a rate such that when it is divided by $\mathrm{N}$, its frequency is equal to $\mathrm{f}_{\mathrm{PFD}}$ and its phase is in sync with the reference clock. This describes a negative feedback mechanism, with the external loop filter components stabilizing the loop and setting the control bandwidth. The $\mathrm{O}$ divider increases the output frequency range by dividing down the VCO output to create more frequency bands than just that of the VCO.

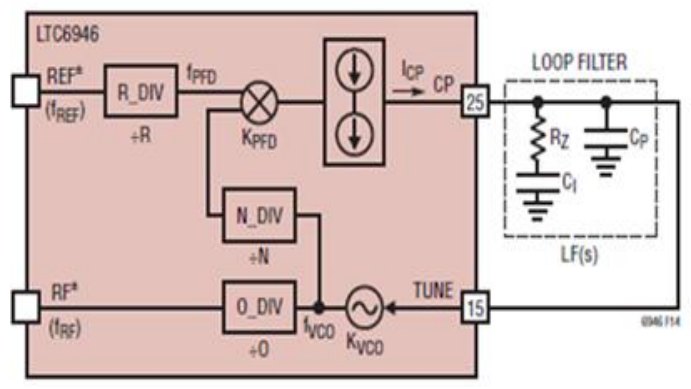

Fig 9: Simplified LTC6946 block diagram [13]

When the loop is locked, the frequency $\mathrm{f}_{\mathrm{VCO}}$ (in $\mathrm{Hz}$ ) produced at the output of the VCO is determined by the reference frequency, $\mathrm{f}_{\mathrm{REF}}$, and the $\mathrm{R}$ and $\mathrm{N}$ divider values, according to:

$\mathrm{f}_{\mathrm{VCO}}=\frac{\mathrm{f}_{\mathrm{REF}} * \mathrm{~N}}{\mathrm{R}}$

The PFD frequency $f_{P F D}$ is given by:

$\mathrm{f}_{\mathrm{PFD}}=\frac{\mathrm{f}_{\mathrm{REF}}}{\mathrm{R}}$

$\mathrm{F}_{\mathrm{VCO}}$ may be alternatively expressed as

$\mathrm{f}_{\mathrm{VCO}}=\mathrm{f}_{\mathrm{PFD}} * \mathrm{~N}$

The output frequency $f_{\text {RF }}$ produced at the output of the $\mathrm{O}$ divider is given by

$\mathrm{f}_{\mathrm{RF}}=\frac{\mathrm{f}_{\mathrm{VCO}}}{0}$

Using the above equations, the output frequency resolution $\mathrm{f}_{\mathrm{STEP}}$ produced by a unit change in $\mathrm{N}$ is given by
$\mathrm{f}_{\mathrm{STEP}}=\frac{\mathrm{f}_{\mathrm{REF}}}{\mathrm{R} * 0}$

Figure 10 shows the setup for measuring the frequency synthesizer board where it is connected to power supplies $5 \mathrm{v}$ and 3.3v, a DC590 controller board connected to PC USB port to operate it under control of PLL wizard software in a personal computer, a reference frequency source and the RF output is connected to the spectrum analyzer. The frequency of the frequency synthesizer is set by the wizard software via the DC590 USB serial controller board. Figure 11 shows the wizard adjusted such that:

- $\quad$ LO frequency band: $1920 \mathrm{MHz}$ to $1980 \mathrm{MHz}$

- Frequency step size (channel-to-channel spacing):20MHz

- $\quad$ Reference clock frequency: $10 \mathrm{MHz}$

The LTC6946 VCO gain is nearly constant as a percentage of the frequency so the loop filter designed at any frequency within the band works for all other frequencies.

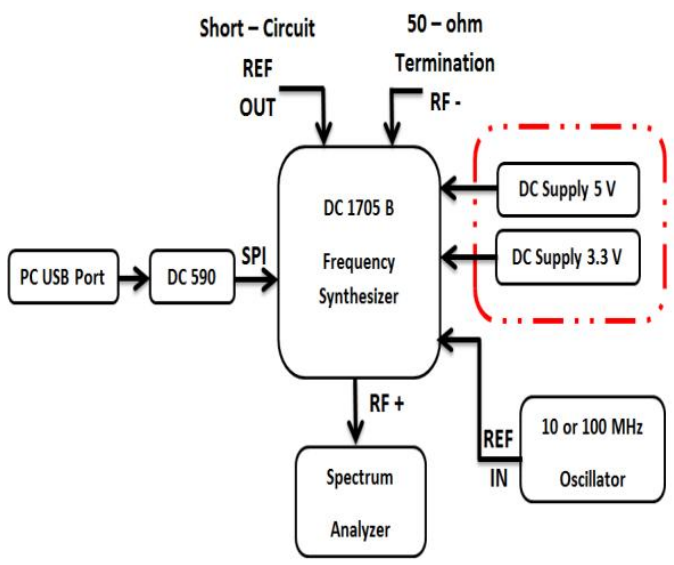

Fig 10: Measurement setup of DC1705B frequency synthesizer

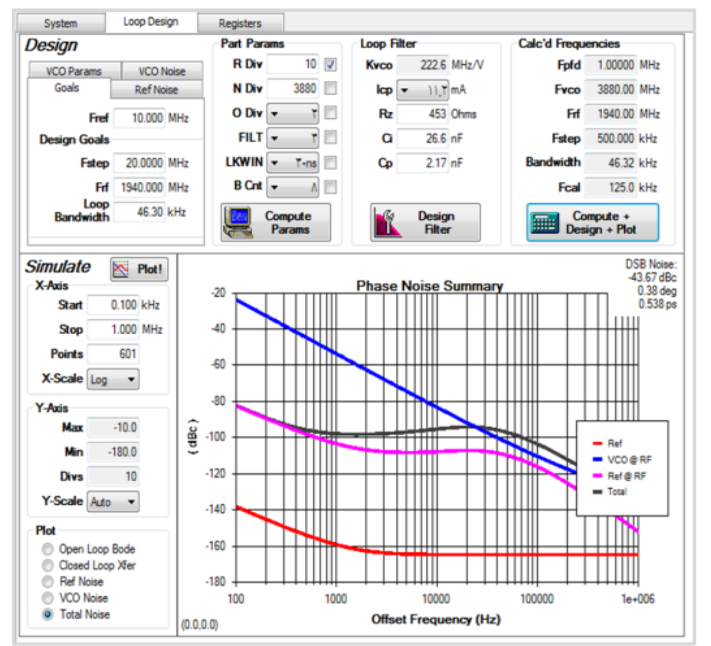

Fig 11: Loop Filter Design for PLLWizard software at frequency $1940 \mathrm{MHZ}$

Figure 12 shows the output of the synthesizer on the screen of the spectrum analyzer. It is clear from the figure that the synthesizer output frequency amounts to $1940 \mathrm{MHz}$ at an output power level of $0 \mathrm{dBm}$. 


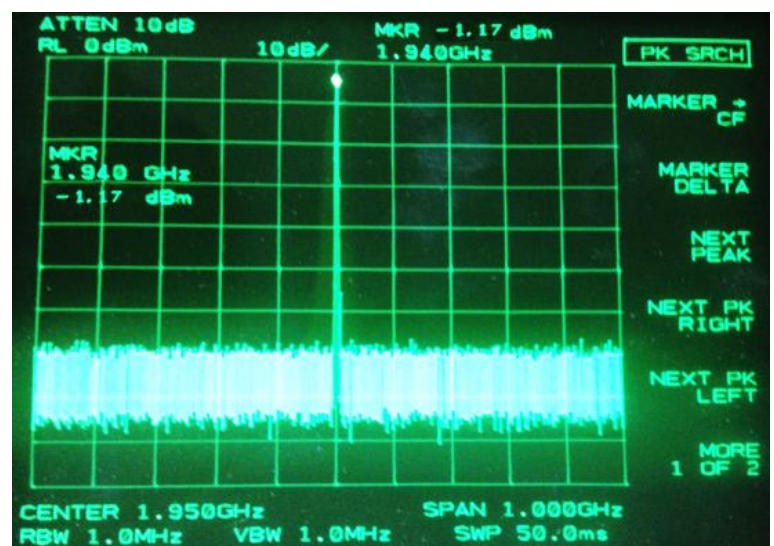

Fig 12: The RF output of the Frequency Synthesizer at frequency $1940 \mathrm{MHz}$

\subsection{The Direct Conversion I/Q Demodulator}

The third and last building block in the active front end is the IQ demodulator for direct frequency conversion, allowing a receiver to demodulate the wide RF signal directly to baseband [14]

The chip LTC5585 is selected to function as a direct conversion quadrature demodulator for the proposed RF receiver. It is suitable for communication receivers where an RF signal is directly converted into I and Q baseband signals with bandwidth of $530 \mathrm{MHz}$ or higher. The LTC5585 incorporates balanced I and Q mixers, LO buffer amplifiers and a precision, high frequency quadrature phase shifter. The integrated on-chip broadband transformer provides a singleended interface at the RF input with a simple off-chip L-C matching. In addition, the LTC5585 provides four analog control voltage interface pins for IIP2 and DC offset correction, greatly simplifying system calibration. The high linearity of the LTC5585 provides excellent spurfree dynamic range of the receiver. These I/Q outputs can interface directly to channel-select filters (LPFs) or to baseband amplifiers.

Demonstration circuit DC1662 is designed for evaluating the LTC5585 IC at RF frequencies from $700 \mathrm{MHz}$ to $3 \mathrm{GHz}$, with a supply voltage range from $0.3 \mathrm{~V}$ to $5.5 \mathrm{~V}$, and about $250 \mathrm{~mA}$ current.

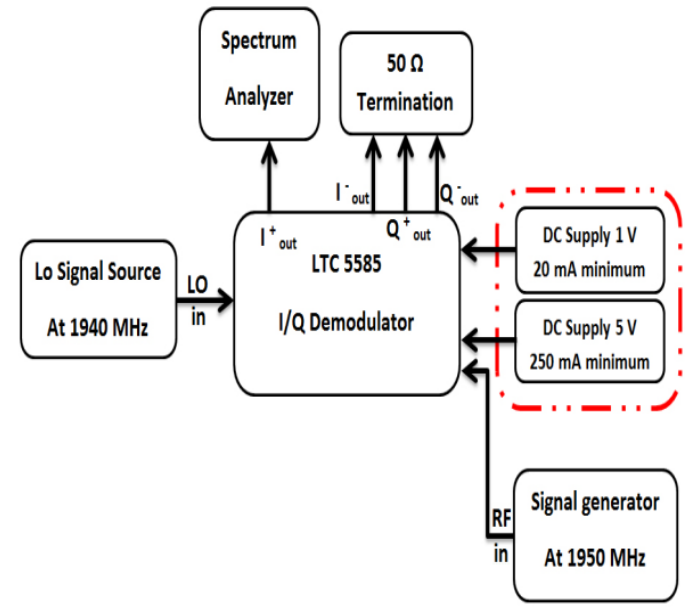

Fig 13: Measurement setup of LTC5585 Demodulator
Figure 13 shows the measuring circuit of the IQ demodulator chip LTC 5585. The output of the LNA is connected to RF $F_{\text {in }}$, the output of the frequency synthesizer is connected to $\mathrm{Lo}_{\text {in }}$ of the IQ demodulator, while a signal analyzer is connected to one baseband channel output of the IQ demodulator and the other three channels matched by $50 \mathrm{Ohm}$. To measure the Baseband output, which has a frequency equals the difference between the two input rf signals of $1950 \mathrm{MHz}$ connected to RFin and 1940MHz connected to Loin.

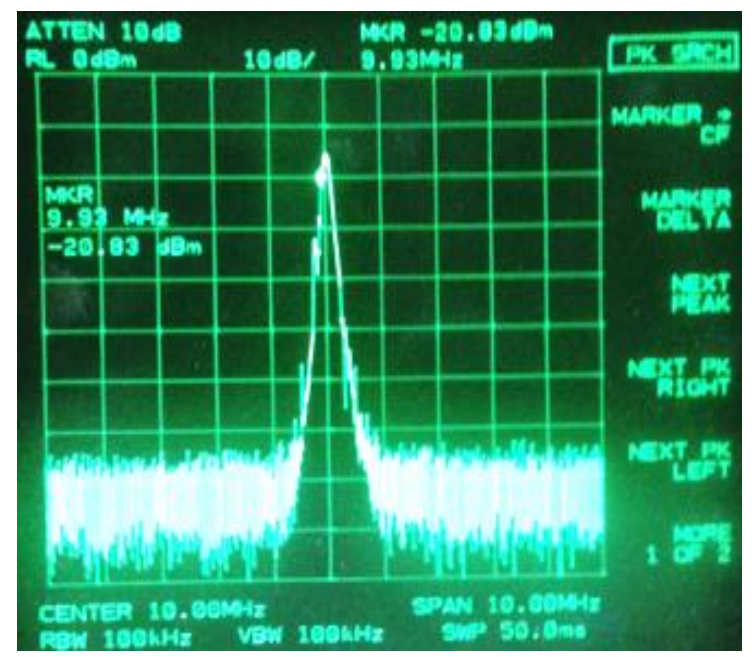

Fig 14: The measured Baseband Signal output of the IQ demodulator

The measured output Baseband signal from the IQ demodulator is depicted in figure 14 , where it has an output frequency of $\left(\mathrm{F}_{\mathrm{RF}}-\mathrm{F}_{\mathrm{LO}}\right)=10 \mathrm{MHz}$ at power level -20.83 $\mathrm{dBm}$.

\section{MEASUREMENTS OF THE WHOLE LTE RF RECEIVER}

The whole receiver is assembled as shown in figure 15 by connecting the tested component together according to the configuration given in figure 1 .

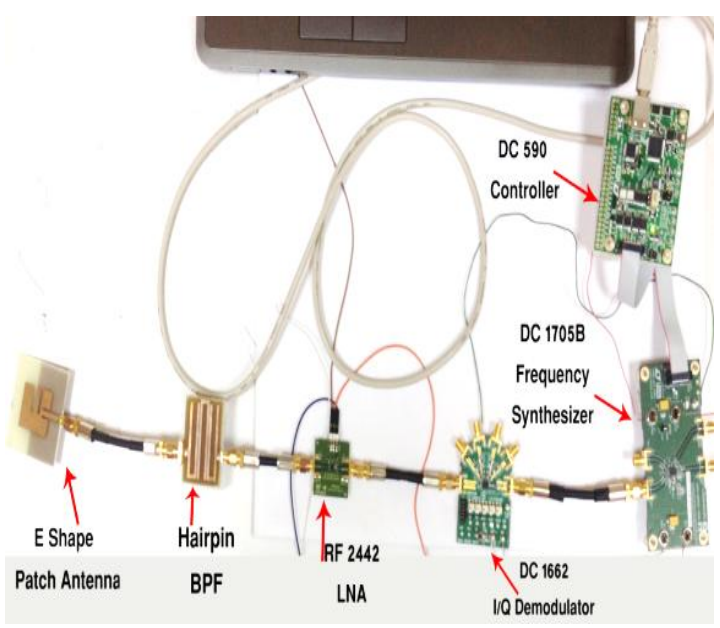

Fig 15: Photo of the proposed RF receiver

Testing setup of the receiver is depicted in Figure 16 the receiver is driven from an rf signal generator to at the level $20 \mathrm{dBm}$ and frequency $1950 \mathrm{MHz}$. A signal analyzer is used to measure the baseband output. 


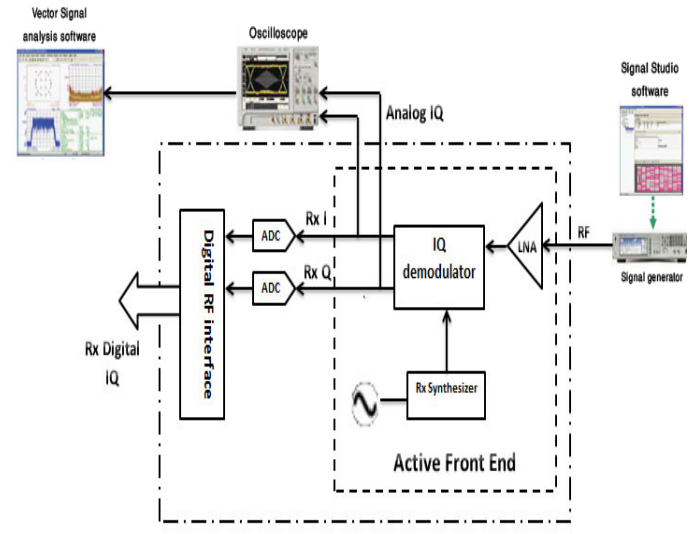

Fig 16: Measurement setups for $\mathrm{RF}$ receiver

Figure 17 displays the input signal to the receiver at a level of $-20 \mathrm{dBm}$ and frequency of $1950 \mathrm{MHz}$, while figure 18 shows the output baseband signal from the receiver at the level of $-62 \mathrm{dBm}$ and frequency $\mathrm{FBB}$ of $10 \mathrm{MHz}$

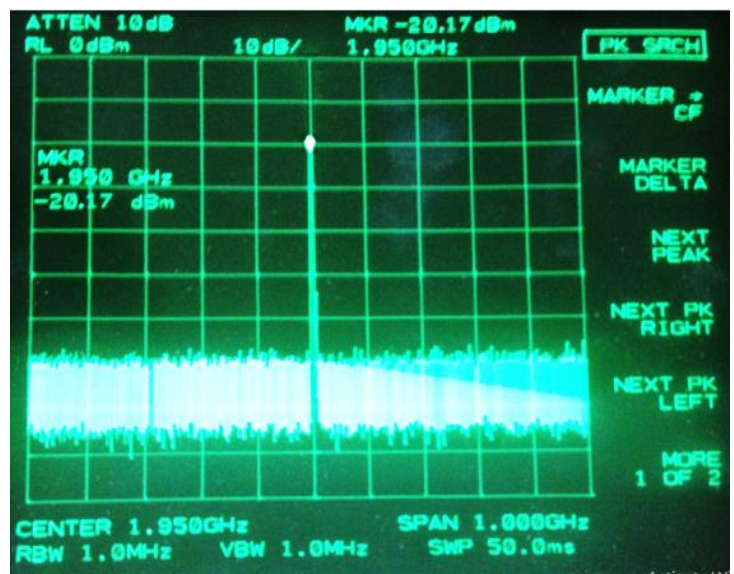

Fig 17: The input signal to the proposed RF receiver 1950 MHz

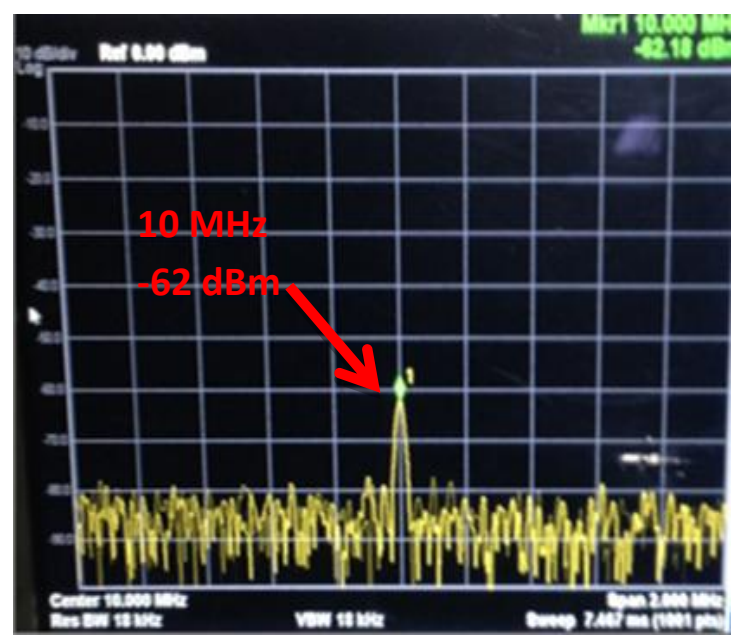

Fig 18: The output baseband signal from the proposed $\mathrm{RF}$ receiver at $10 \mathrm{MHz}$

$$
\begin{aligned}
\mathrm{F}_{\mathrm{BB}} & =\mathrm{F}_{\mathrm{RF}}-\mathrm{F}_{\mathrm{LO}} \\
& =1950 \mathrm{MHz}-1940 \mathrm{MHz}=10 \mathrm{MHz}
\end{aligned}
$$

In addition to testing the functionality of the radio receiver, some other additional performance parameters of the receiver are also determines. The power dissipation amount is 1.65 $\mathrm{W}$. The noise figure NF receiver of the receiver is estimated to be $4.022 \mathrm{~dB}$. The receiver sensitivity $\mathrm{S}$ is also estimated to be $-134.1 \mathrm{dBm}$. Finally the third order intercept point is calculated to be $2.65 \mathrm{dBm}$.

\section{CONCLUSIONS}

An LTE receiver with Zero IF Architecture has been proposed and built from an active part and passive part. The passive components of the receiver are the antenna and filter. The antenna and filter are fabricated on FR4 substrate which is low cost. The size of the proposed antenna with defected ground structure reduced by almost $51 \%$ compared to the size of conventional patch antenna. The bandwidth of the proposed antenna is $150.3 \mathrm{MHz}$. The filter is a third order hairpin band pass filter at resonant frequency $1950 \mathrm{MHz}$ and $3 \mathrm{~dB}$ frequency $90 \mathrm{MHz}$.

The active elements include low noise amplifier, I/Q demodulator and frequency synthesizer. They are selected from off-shelf components after an intensive search to satisfy the requirements of the front end LTE receiver.

RF low noise amplifier (RF2442) Low Noise and High Intercept Point, $500 \mathrm{MHz}$ to $2500 \mathrm{MHz}$ Operation, at the operating frequency $1950 \mathrm{MHz}$ and $\mathrm{Vcc}=3.6 \mathrm{~V}$. Noise Figure $1.6 \mathrm{~dB}$, achieve gain of about $12 \mathrm{~dB}$, Input return loss -15 $\mathrm{dB}$, output return loss $-13 \mathrm{~dB}$, reverse isolation $-20 \mathrm{~dB}$.

The frequency synthesizer (LTC6946) operates at frequency range $0.37 \mathrm{GHz}$ to $5.7 \mathrm{GHz}$, so that it covers all LTE bands. LTC6946 is programed using DC 590 controller board and PLL wizard software which is set to the operating frequency $1950 \mathrm{MHz}$

By the direct conversion I/Q demodulator (LTC5585) the RF signal is directly converted into I and Q baseband signals at $10 \mathrm{MHz}$. Finally the whole LTE receiver is assembled together and tested. It is found that the parameters of the receiver can satisfy the requirements of the LTE front end for the user equipment. This work succeeded to design, build and test a fully functioning LTE receiver for the user equipment.

In the future work the receiver will be integrated in a single PCB board.

\section{REFERENCES}

[1] 3GPP TS 36.101, "EUTRA User Equipment Radio Transmission and Reception", September 2008, vol. 8.3.0.

[2] Maode Ma, Senior Member, IEEE Hui Li Member, and Yueyu Zhang Jin Cao, "A Survey on Security Aspects for LTE and LTE-A Networks", 2013.

[3] Erik Dahlman, Stefan Parkvall, and Johan Sköld, 4G LTE/LTE-Advanced for Mobile Broadband, May 2011.

[4] Sivonen P, Tervaluoto J, Mikkola N, et al. A 1.2-V RF front-end with on-chip VCO for PCS 1900 direct conversion receiver in $0.13 \mu \mathrm{m}$ CMOS. IEEE J SolidState Circuits, 2006, 41(2): 384

[5] Wang Riyan, Huang Jiwei, Li Zhengping, Zhang Weifeng, and Zeng Longyue, Journal of Semiconductors, A 1.2-V CMOS front-end for LTE direct conversion SAW-less receiver, Vol. 33, No. 3 , March 2012. 
[6] Rodrigures S, Rusu A, Ismail M. WiMAX/LTE receiver front-end in $90 \mathrm{NM}$ CMOS. IEEE International Symposium on Circuit and System (ISCAS), 2009: 1036

[7] D. A. L. D. S. C. Dr. Lisimachos P. Kondi, 4G Wireless Video Communications, Dr. Haohong Wang, 17 JUN 2009.

[8] Dalia Sadek, Abdelhalim Zekry,and Heba A. Shawkey," Passive Front End for LTE TDD Transceiver ", International Journal of Computer Applications, (09758887), vol.116-No.8, pp. 6-11 April, 2015.

[9] G. Gonzalez, Microwave Transistor Amplifiers, Prentice Hall, 1984.

[10] LNA Design Using Spectra RF Application Note Product Version 5.0, by Cadence Design Systems., December 2003.
[11] P. Vizmuller. RF Design Guide: Systems, Circuits and Equations. Artech House, 1995.

[12] F. M. Gardner, Phaselock Techniques Hardcover, July 29, 2005.

[13] [Online].Available:http://cds.linear.com/docs/en/datashe et/6946fb.pdf.

[14] 1. S. Maas, Microwave Mixers, Artech House, Norwood, MA, 1986.

[15] Vinay M M, Roy Paily and Anil Mahanta," 2013 26th International Conference on VLSI Design and the 12th International Conference on Embedded Systems" "Gain, NF and IIP3 Budgeting of LTE Receiver Front End"

[16] Marwa Mansour, Abdelhalim Zekry, and R.S. Ghoname ," Building Radio Frequency Transmitter for LTE User Equipment", Foundation of Computer Science FCS, July 2015 . 